\title{
Positives and negatives of nonsteroidal anti- inflammatory drugs in bone healing: the effects of these drugs on bone repair
}

This article was published in the following Dove Press journal:

Drug Design, Development and Therapy

\author{
Barbara Lisowska' \\ Dariusz Kosson ${ }^{2}$ \\ Karolina Domaracka ${ }^{3}$ \\ 'Department of Anesthesiology \\ and Intensive Care, John Paul II \\ Western Hospital, Grodzisk \\ Mazowiecki, Poland; ${ }^{2}$ Department \\ of Anaesthesiology and Intensive \\ Care, Medical University of Warsaw, \\ Division of Teaching Warsaw, \\ Warsaw, Poland; ${ }^{3}$ Department of \\ Anaesthesiology and Intensive \\ Care, Medical University of Warsaw, \\ Warsaw, Poland
}

\begin{abstract}
Tissue damage following injury triggers the processes of coagulation, inflammation and healing. In tissues surrounding the bone, the result of the healing process is a scar, while bone tissue has a unique ability to achieve shape, strength and pre-injury function. Bone healing is a process of regeneration rather than classic recovery. The result of this process is the formation of new, healthy bone tissue instead of a scar. Many factors can inhibit or impair the bone healing process, and their influence is critical during the stages of inflammation and angiogenesis and finally on the clinical outcome. Nonsteroidal anti-inflammatory drugs (NSAIDs) play an essential role associated with their analgesic potency and anti-inflammatory effects. NSAIDs are also the most often used drugs in patients who require pain control and inflammation reduction due to musculoskeletal diseases or injures. Although their analgesic effect is well documented, NSAIDs also interfere with bone healing; therefore, the relative benefits and disadvantages connected with their administration should be taken into consideration. Despite the negative effect, NSAIDs have beneficial properties, but their clinical benefits in relation to dose and time of use are still unclear. Therefore, in this review, we focus on bone healing with relation to the impact of NSAIDs.
\end{abstract}

Keywords: bone healing, NSAIDs, negative, positive effect

\section{Introduction}

Tissue damage following injury triggers the processes of coagulation, inflammation and healing. In tissues surrounding the bone, the result of the healing process is a scar, while bone tissue has a unique ability to achieve shape, strength and pre-injury function. Bone healing is a process of regeneration rather than classic recovery. The result of this process is the formation of a new, healthy bone tissue instead of a scar.

The course of bone healing includes bone formation, angiogenesis and soft tissue healing, and can be affected by many factors depending on the degree of disorders. Among these factors, non-steroidal anti-inflammatory drugs (NSAIDs) can inhibit or impair bone healing process because their influence is critical on the stages of healing including inflammation, coagulation and angiogenesis and finally on the clinical outcome. The mechanism of action of NSAIDs is associated with the inhibition of cyclooxygenase (COX) enzyme activity and prostanoid pathway which is responsible for the synthesis of prostanoids by biotransformation of arachidonic acid released from the phospholipids of cell membranes by phospholipase A2.

Two forms of COX enzyme are isolated: COX-1 and COX-2. COX-1 is expressed in most cells and takes part in the synthesis of prostaglandins under physiological
Correspondence: Dariusz Kosson Department of Anesthesiology and Intensive Care, Medical University of Warsaw, Division of Teaching Warsaw, 4 Lindley Street, 02-005 Warsaw, Poland Email kosson@wp.pl

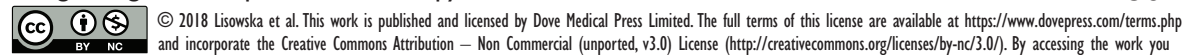

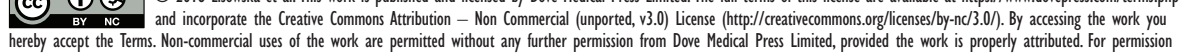

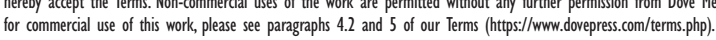


conditions. The synthesis of COX-2 is stimulated by an inflammatory state, and COX-2 induces the release of prostaglandins in response to inflammation. The inhibition of COX isoenzymes activity by NSAIDs leads to the decrease in prostanoids synthesis; among these prostanoids, prostaglandins play a crucial role in inflammatory response.

NSAIDs can be divided into three main groups: classic, selective and coxibs. The classic drugs have been traditionally thought to act inhibiting the activity of both COX-1 and COX-2 with near-equal potency in contrast to selective COX-2 inhibitors, which preferentially inhibit the activity of COX-2 while inhibiting COX-1 with less potency, and highly selective inhibitors of the COX-2 isoenzyme, coxibs. It is worth highlighting that the outcome of treatment with the inhibitors of COX isoenzymes also depends on the side effects connected with their administration.

NSAIDs play an essential role associated with their analgesic potency and anti-inflammatory effects. NSAIDs are also the most often used drugs in patients who require pain control and inflammation reduction due to musculoskeletal diseases or injures. Although their analgesic effect is well documented, NSAIDs also interfere with bone healing; therefore, the relative benefits and disadvantages connected with their administration should be taken into consideration.

\section{Bone response to trauma}

Bone trauma triggers bone healing involving the processes of coagulation and inflammatory response. In tissues surrounding the bone, the result of healing process is a scar, while bone tissue has a unique ability to achieve shape, strength and preinjury function. Bone healing is the process of regeneration rather than classic recovery. The result of this process is the formation of a new, healthy tissue instead of a scar.

At the site of a bone injury, between the bone fragments, forms a hematoma in which the stages of coagulation, inflammatory response and healing take place. The early phase is characterized by the hematoma having high concentrations of mature granulocytes and monocytes/macrophages, as well as helper and cytotoxic lymphocytes. In addition, high levels of inflammatory and anti-inflammatory cytokines are found in the hematoma. ${ }^{1}$

A previous study showed that in the immediate postoperative period following alloplastic knee surgery, IL-6, TGF and IL-8 concentrations in blood drainage from the wound were several times higher than those in serum, which were determined at the same time, confirming the high intensity of the humoral response to injury. ${ }^{2}$ The cytokines present in the hematoma, as well as other growth factors, stimulate the differentiation of mesenchymal cells of the bone marrow toward the chondrogenic and osteogenic lines.

The next stage of the bone healing process is the formation of soft (young) and hard (calcareous) callus.

The quality and effectiveness of the body's response to an injury depend on its general condition, which is an exponent of its defensive and regenerative abilities, as well as the type and size of the injury. Among the factors affecting bone healing, local and systemic factors such as the degree and extent of injury, cardiovascular disorders, age, gender, chronic diseases, nutritional status, stimulants and drugs used should be mentioned. ${ }^{3-5}$ For example, in the study of Jeffcoach et al, complications in the healing of long bone fractures occurred two to three times more frequently among smokers and patients taking NSAIDs than in the control group. ${ }^{6}$

\section{Effects of NSAIDs on bone healing}

Drugs are the most controllable factor because their impact on bone healing can be taken into account with respect to their choice and dosage. This especially applies to drugs administered due to an injury, such as analgesic drugs, among which the NSAIDs are at the forefront in terms of frequency of use. The benefits of using NSAIDs are primarily due to their analgesic efficacy and anti-inflammatory effect, which are especially important for patients with various forms of arthritis because these drugs reduce disease activity and improve their quality of life. ${ }^{7}$ Taking into account the pharmacodynamics of NSAIDs, it should be noted that they affect all stages of bone healing involving the bone and surrounding tissues.

It is not surprising therefore that many publications present studies evaluating the effects of NSAIDs divided into three main groups: classic, selective and coxibs. Studies have been conducted on both human and animal materials, in vitro and in vivo. Indomethacin, piroxicam, naproxen and celecoxib were the drugs most commonly reported in studies. Based on the obtained results, most authors negatively assessed the effect of NSAIDs on bone healing. However, the presence of publications in which authors present positive aspects of using NSAIDs after bone injuries should be emphasized.

\section{The negative effects of NSAIDs}

The negative effect of NSAIDs seems to be mostly related to the COX-2 blockade, as indicated by the results of comparative studies in animals without the COX-1 gene in which no significant bone healing disturbances have been 
demonstrated, whereas in animals with the COX-2 blockade, worse bone union has been reported. It is not entirely clear how COX-2 works, but the research of Simon et al has confirmed that COX-2 is necessary for mesenchymal cells to differentiate into osteoblasts during fracture healing. ${ }^{8}$ Although taking into account the half-life of the investigated drugs, Aspenberg raised doubts about the validity of the interpretation described by Simon et al. ${ }^{9}$

This does not change the fact that other studies on COX-2 expression have also confirmed the negative effect of celecoxib on the healing process by limiting osteogenesis. ${ }^{10-12}$ In light of the presented facts, the effect of COX-2 inhibitors is of particular importance for healing disorders such as delayed union or nonunion. The results presented by Murnaghan et al also confirmed an inhibition of angiogenesis in the fracture gap due to NSAID administration. The authors suggested a connection between angiogenesis impairment and bone healing delay. ${ }^{13}$ In turn, Yoon et al demonstrated a decrease in the osteogenic potential of mesenchymal stem cells by high doses of COX-2 blockers in the presence of inflammation. ${ }^{14}$

Comparative studies on the effects of indomethacin, ketorolac, piroxicam, diclofenac and celecoxib on the proliferation, toxicity and apoptosis of human osteoblasts in vitro showed that all of these drugs limited their proliferation by inhibiting the transition from G0 phase to G1, that is, from rest to metabolic activity, while only celecoxib induced cell apoptosis. Since the supply of prostaglandins did not reverse the described changes in the osteoblast culture and, at the same time, changes in mRNA and in the expression of p27 (kip1) and cyclin D2 and p-cdk2 proteins in osteoblasts have been reported, the authors have concluded that NSAIDs have other mechanisms of action. ${ }^{15}$

This is confirmed by Nagano et al, ${ }^{16}$ based on the example of celecoxib, which, in addition to the COX-2 blockade, also inhibits the genes produced by the transcriptional Wnt/-catenin canon signaling pathway. The Wnt/ $\beta$-catenin signaling pathway plays a key role in the differentiation and proliferation of osteoblast precursor cells by blocking osteoclastogenesis. ${ }^{16}$

Retrospective studies in large patient groups have confirmed that long-term use of aspirin and NSAIDs doubles the risk of healing disorders. It is worth emphasizing that short treatment (up to 7 days) delayed healing while prolonged treatment resulted in nonunion. ${ }^{17}$ Healing disorders and increased risk of nonunion or delayed union after treatment with both COX-1 and COX-2 inhibitors have also been reported in the observations of Burd et al and Giannoudis et al, based on retrospective studies of patients after injury. ${ }^{18,19}$ Similar conclusions regarding the NSAID treatment of patients after surgery were presented by Sagi et al, who proposed to reduce the use of NSAIDs to no more than 1 week, confirming the relationship between the duration of the treatment and nonunion. According to the authors, it would reduce the risk of heterotopic ossification without deterioration in healing. ${ }^{20}$

More restrictive recommendations were presented by Marquez-Lara et al, who suggested the replacement of NSAIDs by opioids in analgesic treatment. ${ }^{21}$ Although it should be emphasized that the influence of opioids on fracture healing is still uncertain, a few publications have shown a relationship between higher risk of fracture nonunion or reduced bone mineral density and opioid treatment. ${ }^{22,23}$

For example, Chrastil et $\mathrm{al}^{23}$ found a decrease in callus strength in morphine-treated animals compared with control, saline-treated group. Their study identified that morphine treatment impairs fracture healing and in consequence leads to weaker callus. These observations can cause a change in opinion about morphine safety in orthopedic population. In contrast to the study of Chrastil et al, Janas and Folwarczna showed the beneficial effect of opioid analgesics on the skeletal system, especially in animals with estrogen deficiency. ${ }^{24}$

The effect of NSAIDs on adipogenesis, at the expense of osteogenesis inhibition, was described by Kellinsalmi et al, who evaluated the effect of indomethacin and parecoxib on human osteoblast and osteoclast activity in vitro. ${ }^{25}$ The results confirmed the inhibition of differentiation of both cell lines by $90 \%$ and the increase in the number of adipocytes in the presence of indomethacin and parecoxib compared to the control group.

A positive aspect of the action of the NSAIDs is the stimulation of collagen synthesis, which, as should be expected, could improve healing in the tissues surrounding the fracture. In turn, the study by Hadjicharalambous et al confirmed the stimulating effect of NSAIDs on the stem cells derived from adipose tissue, in their differentiation toward the osteogenic line. According to the researchers, meloxicam and parecoxib showed the most beneficial effect. ${ }^{26}$

\section{The positive effects of NSAIDs}

The beneficial effects of NSAIDs use are not limited to analgesic and anti-inflammatory ones. Inhibition of prostaglandin synthesis may also contribute to the limitation of pathological bone growth and the formation of heterotopic ossification, which may be of particular importance after hip surgery. ${ }^{27,28}$ 
Huub et al have shown a comparable effect of an NSAID (indomethacin and rofecoxib) on the restriction of heterotopic ossification after alloplastic hip surgery. ${ }^{29}$ Likewise, other authors have shown similar effects in the prevention of heterotopic ossification with diclofenac. ${ }^{30,31}$

In turn, Driban et al have presented the results of their research indicating an attenuation of inflammation and early articular cartilage degeneration of hand joints in ibuprofentreated rats. The obtained results have also confirmed a reduction of IL-1 and IL-6 concentrations during ibuprofen administration. $^{32}$

The reduction of cytokine concentration during ibuprofen treatment has been shown by Jain et al, who focused their study on the impact of ibuprofen on trabecular structure in forearm bones of rats. ${ }^{33}$ According to the obtained results, ibuprofen treatment was able to improve trabecular bone quality by reducing osteoclasts and bone inflammatory cytokines (IL- $1 \beta$ and TNF- $\alpha$ ). The presented evidence can confirm that systemic treatment with ibuprofen contributes to the reduction of inflammation and bone catabolism, which in turn may aid bone healing.

\section{NSAIDs and angiogenesis}

Another point of action of NSAIDs is their effect on angiogenesis. Angiogenesis is crucial for both the preservation of bone tissue homeostasis and the response to injury. Angiogenic factors are synthesized both in endothelial cells and in bone tissue cells, so inhibiting their synthesis and release may contribute to slowing down the healing process. ${ }^{34}$ Animal studies have confirmed that the temporary inhibition of VEGFR-1 and VEGFR-2 expression by meloxicam correlated with the time of its administration, but no difference was found in VEGF expression compared to the control group. ${ }^{35}$

VEGF is one of the most important growth factors in angiogenesis, so the effect on its expression may interfere with the development of the vessel between the fracture gaps and thus delay union. Restriction of VEGF expression disrupts communication between vessels and osteoblasts, which are the main source of VEGF. ${ }^{36,37}$

The effect of NSAIDs on angiogenesis is ambiguous. For example, Lu et $\mathrm{al}^{38}$ demonstrated a positive effect of NSAIDs on vascular density in the fracture gap compared to the control group. The study also showed bone healing disorders in the form of osteo- and chondrogenesis inhibition. Anti-inflammatory activity of NSAIDs has been shown to decrease IL-1 levels, but no effect of NSAIDs on macrophage and neutrophil migration has been demonstrated. ${ }^{38}$

\section{NSAIDs and tendon healing}

Contrary to the effects on bone, NSAIDs seem to have a positive effect on soft tissue healing by stimulating collagen synthesis, which is also reflected in improved endurance of the stiffened tendons. However, the presented opinions are not consistent because, contrary to appearances, celecoxib and indomethacin have been shown to inhibit enthesis healing and weaken the endurance of the Achilles tendon in tested animals.

Similar results have been reported by other authors confirming the harmful effects of NSAIDs on the biomechanical properties of damaged tendons, which were related to deterioration in their healing, ${ }^{39}$ and even, according to Ferry et al, with a reduction in collagen content, although these authors did not confirm the negative effects of ibuprofen. ${ }^{40}$

The difficulty in obtaining a clear opinion is indicated by the results of Schwarting et al. Based on an in vitro model, the authors confirmed the negative effect of NSAIDs (ibuprofen, parecoxib) on bone union, whereas in the case of tendons they did not achieve an unequivocal answer, indicating the need for further studies. ${ }^{41}$

The inhibition of migration and proliferation of tendon cells by NSAIDs has been demonstrated by Tsai et al, who did not confirm the effects of COX-2 (celecoxib) inhibitors on the expression of collagen I and II. ${ }^{42,43}$ Indomethacin has been shown to have negative effects on the proliferation of human tenocyte cultures. ${ }^{44}$

In animals treated with indomethacin or parecoxib, the quality of the Achilles tendon was lower in terms of tensile strength compared to the control group. ${ }^{45}$ The effect of the duration of NSAIDs use on healing of tendons has also been confirmed; according to Connizzo et al, the use of indomethacin for the first 7 days after surgery contributed to the deterioration of healing while later treatment was irrelevant. ${ }^{46}$

Interesting results were presented by Oak et al for the simultaneous inhibition of 5-lipoxygenase (5-LOX) and COX pathways. Similar to COX pathway, arachidonic acid is also the substrate for 5-LOX pathway. Unlike the COX pathway, in the 5-LOX enzyme pathway, leukotrienes are produced which also contribute to the inflammatory response. Based on the results obtained, the authors have concluded that simultaneous inhibition of the COX and 5-LOX pathways contributed to the improvement in posttraumatic tendon regeneration. ${ }^{47}$

While the use of NSAIDs after injury in the context of tendon healing is controversial, and should rather be avoided in the immediate posttraumatic period, long-term treatment 
with NSAIDs did not affect the elasticity and strength of the tendons as assessed by their response to effort. ${ }^{48}$

\section{Conclusion}

This review showed not only negative but also slightly positive effects of NSAIDs on the healing process of bone and surrounding tissues. So the question remains, why is the use of NSAIDs so persistently popular, despite the defects described above. The answer seems to be their popularity enhanced by advertising, their undoubted analgesic efficacy (without the scary symptoms accompanying the use of opioids) and partial lack of translation of the reported results into the occurrence of symptoms over a period which could be associated with NSAID use. For instance, the loosening of prostheses has been confirmed in patients taking NSAIDs, but it does not affect all of them and it happens over such a long time that it is difficult to convince the patients and medical staff that it is a plausible correlation between the complications and NSAID therapy. Therefore, it seems reasonable, and realistic proposal would be to recommend limiting the use of NSAIDs at the time of their greatest negative impact, that is, the first few days after the injury.

\section{Disclosure}

The authors report no conflicts of interest in this work.

\section{References}

1. Hoff P, Gaber T, Strehl C, et al. Immunological characterization of the early human fracture hematoma. Immunol Res. 2016;64(5-6): 1195-1206.

2. Lisowska B. The influence of stress surgery on immunological parameters in patients with osteoarthritis and rheumatoid arthritis. Reumatologia. 2008;46(Suppl 1):1-69.

3. Pountos I, Georgouli T, Calori GM, Giannoudis PV. Do nonsteroidal anti-inflammatory drugs affect bone healing? A critical analysis. ScientificWorldJournal. 2012;2012:606404.

4. Gaston MS, Simpson AHRW. Inhibition of fracture healing. J Bone Joint Surg Br. 2007;89(12):1553-1556.

5. Risto O, Hammar E, Hammar K, Fredrikson M, Hammar M, Wahlström O Elderly men with a history of distal radius fracture have significantly lower calcaneal bone density and free androgen index than age-matched controls. Aging Male. 2012;15(1):59-62.

6. Jeffcoach DR, Sams VG, Lawson CM, et al. Nonsteroidal anti-inflammatory drugs' impact on nonunion and infection rates in long-bone fractures. J Trauma Acute Care Surg. 2014;76(3):779-783.

7. Kroon FP, van der Burg LR, Ramiro S, et al. Non-steroidal antiinflammatory drugs (NSAIDs) for axial spondyloarthritis (ankylosing spondylitis and non-radiographic axial spondyloarthritis). Cochrane Database Syst Rev. 2015;(7):CD010952.

8. Simon AM, Manigrasso MB, O'Connor JP. Cyclo-oxygenase 2 function is essential for bone fracture healing. J Bone Miner Res. 2002;17(6): 963-976.

9. Aspenberg P. Differential inhibition of fracture healing by non-selective and cyclooxygenase-2 selective non-steroidal anti-inflammatory drugs. J Orthop Res. 2004;22(3):684.
10. Gerstenfeld LC, Thiede M, Seibert K, et al. Differential inhibition of fracture healing by non-selective and cyclooxygenase-2 selective non-steroidal anti-inflammatory drugs. J Orthop Res. 2003;21(4): 670-675.

11. Daluiski A, Ramsey KE, Shi Y, et al. Cyclooxygenase-2 inhibitors in human skeletal fracture healing. Orthopedics. 2006;29(3):259-261.

12. Herbenick MA, Sprott D, Stills H, Lawless M. Effects of a cyclooxygenase 2 inhibitor on fracture healing in a rat model. Am J Orthop (Belle Mead NJ). 2008;37(7):E133-E137.

13. Murnaghan M, Li G, Marsh DR. Nonsteroidal anti-inflammatory druginduced fracture nonunion: an inhibition of angiogenesis? J Bone Joint Surg Am. 2006;88(Suppl 3):140-147.

14. Yoon DS, Yoo JH, Kim YH, Paik S, Han CD, Lee JW. The effects of COX-2 inhibitor during osteogenic differentiation of bone marrowderived human mesenchymal stem cells. Stem Cells Dev. 2010;19(10): $1523-1533$.

15. Chang JK, Li CJ, Liao HJ, Wang CK, Wang GJ, Ho ML. Anti-inflammatory drugs suppress proliferation and induce apoptosis through altering expressions of cell cycle regulators and pro-apoptotic factors in cultured human osteoblasts. Toxicology. 2009;258(2-3):148-156.

16. Nagano A, Arioka M, Takahashi-Yanaga F, Matsuzaki E, Sasaguri T. Celecoxib inhibits osteoblast maturation by suppressing the expression of Wnt target genes. J Pharmacol Sci. 2017;133(1):18-24.

17. Mills L, Tsang J, Hopper G, Keenan G, Simpson AH. The multifactorial aetiology of fracture nonunion and the importance of searching for latent infection. Bone Joint Res. 2016;5(10):512-519.

18. Burd TA, Hughes MS, Anglen JO. Heterotopic ossification prophylaxis with indomethacin increases the risk of long-bone nonunion. $J$ Bone Joint Surg Br. 2003;85(5):700-705.

19. Giannoudis PV, MacDonald DA, Matthews SJ, et al. Nonunion of the femoral diaphysis: the influence of reaming and non-steroidal antiinflammatory drugs. J Bone Joint Surg Br. 2000;82(5):655-658.

20. Sagi HC, Jordan CJ, Barei DP, Serrano-Riera R, Steverson B. Indomethacin prophylaxis for heterotopic ossification after acetabular fracture surgery increases the risk for nonunion of the posterior wall. J Orthop Trauma. 2014;28(7):377-383.

21. Marquez-Lara A, Hutchinson ID, Nuñez F Jr, Smith TL, Miller AN. Nonsteroidal anti-inflammatory drugs and bone-healing: a systematic review of research quality. JBJS Rev. 2016;4(3):pii: 01874474-201603000-00005.

22. Grey A, Rix-Trott K, Horne A, Gamble G, Bolland M, Reid IR. Decreased bone density in men on methadone maintenance therapy. Addiction. 2011;106:349-354.

23. Chrastil J, Sampson C, Jones KB, Higgins TF. Postoperative opioid administration inhibits bone healing in an animal model. Clin Orthop Relat Res. 2013;471(12):4076-4081.

24. Janas A, Folwarczna J. Opioid receptor agonist may favorable bone mechanical prosperities in rats with estrogen efficiency induced osteoporosis. Naunyn Schmiedebergs Arch Pharmacol. 2017;390(2): $175-185$.

25. Kellinsalmi M, Parikka V, Risteli J, et al. Inhibition of cyclooxygenase-2 down-regulates osteoclast and osteoblast differentiation and favours adipocyte formation in vitro. Eur J Pharmacol. 2007;572(2-3):102-110.

26. Hadjicharalambous C, Alexaki VI, Alpantaki K. Effects of NSAIDs on the osteogenic differentiation of human adipose tissue-derived stromal cells. J Pharm Pharmacol. 2016;68(11):1403-1408.

27. VuolteenahoK, Moilanen T, Moilanen E. Non-steroidal anti-inflammatory drugs, cyclooxygenase-2 and the bone healing process. Basic Clin Pharmacol Toxicol. 2008;102(1):10-14.

28. Fransen M, Neal B. Non-steroidal anti-inflammatory drugs for preventing heterotopic bone formation after hip arthroplasty. Cochrane Database Syst Rev. 2004;(3):CD001160.

29. Huub JL, Van Der Heide WJ, Van Sorge A, Van Kampen A, Schreurs W. Similar effects of rofecoxib and indomethacin on the incidence of heterotopic ossification after hip arthroplasty. Acta Orthop. 2007;78(1):90-94.

30. Wahlström O, Risto O, Djerf K, Hammerby S. Heterotopic bone formation prevented by diclofenac: prospective study of $100 \mathrm{hip}$ arthroplasties. Acta Orthop Scand. 1991;62(5):419-421. 
31. Sell S, Phillips O, Handel M. No difference between two doses of diclofenac in prophylaxis of heterotopic ossifications after total hip arthroplasty. Acta Orthop Scand. 2004;75(1):45-49.

32. Driban JB, Barr AE, Amin M, Sitler MR, Barbe MF. Joint inflammation and early degeneration induced by high force reaching are attenuated by ibuprofen in an animal model of work-related musculoskeletal disorder. J Biomed Biotechnol. 2011;2011:691412.

33. Jain NX, Barr-Gillespie AE, Clark BD, et al. Bone loss from high repetitive high force loading is prevented by ibuprofen treatment. J Musculoskelet Neuronal Interact. 2014;14(1):78-94.

34. Chim SM, Tickner J, Chow ST, et al. Angiogenic factors in bone local environment. Cytokine Growth Factor Rev. 2013;24(3):297-310.

35. Arantes RV, Cestari TM, Viscelli BA, et al. Meloxicam temporally inhibits the expression of vascular endothelial growth factor receptor (VEGFR)-1 and VEGFR-2 during alveolar bone repair in rats. J Periodontol. 2015;86(1):162-172.

36. $\mathrm{Hu} \mathrm{K}$, Olsen BR. The roles of vascular endothelial growth factor in bone repair and regeneration. Bone. 2016;91:30-38.

37. Hu K, Olsen BR. Vascular endothelial growth factor control mechanisms in skeletal growth and repair. Dev Dyn. 2017;246(4):227-234.

38. Lu C, Xing Z, Wang X, Mao J, Marcucio RS, Miclau T. Anti-inflammatory treatment increases angiogenesis during early fracture healing. Arch Orthop Trauma Surg. 2012;132(8):1205-1213.

39. Cohen DB, Kawamura S, Ehteshami JR, Rodeo SA. Indomethacin and celecoxib impair rotator cuff tendon-to-bone healing. Am J Sports Med. 2006;34:362-369.

40. Ferry ST, Dahners LE, Afshari HM, Weinhold PS. The effects of common anti-inflammatory drugs on the healing rat patellar tendon. Am J Sports Med. 2007;35(8):1326-1333.
41. Schwarting T, Pretzsch S, Debus F, Ruchholtz S, Lechler F. The effect of cyclooxygenase inhibition on tendon-bone healing in an in vitro coculture model. Mediators Inflamm. 2015;2015:10(926369).

42. Tsai WC, Hsu CC, Chen CP, Chen MJ, Lin MS, Pang JH. Ibuprofen inhibition of tendon cell migration and down-regulation of paxillin expression. J Orthop Res. 2006;24(3):551-558.

43. Tsai WC, Hsu CC, Chou SW, Chung CY, Chen J, Pang JH. Effects of celecoxib on migration, proliferation and collagen expression of tendon cells. Connect Tissue Res. 2007;48(1):46-51.

44. Mallick E, Scutt N, Scutt A, Rolf C. Passage and concentrationdependent effects of indomethacin on tendon derived cells. J Orthop Surg Res. 2009;4:9.

45. Dimmen S, Nordsletten L, Engebretsen L, Steen H, Madsen JE. The effect of parecoxib and indometacin on tendon-to-bone healing in a bone tunnel: an experimental study in rats. J Bone Joint Surg Br. 2009; 91(2):259-263.

46. Connizzo BK, Yannascoli SM, Tucker JJ, et al. The detrimental effects of systemic ibuprofen delivery on tendon healing are time-dependent. Clin Orthop Relat Res. 2014;472(8):2433-2439.

47. Oak NR, Gumucio JP, Flood MD, et al. Inhibition of 5-LOX, COX-1, and COX-2 increases tendon healing and reduces muscle fibrosis and lipid accumulation after rotator cuff repair. Am J Sports Med. 2014;42(12): 2860-2868.

48. Rooney SI, Baskin R, Torino DJ, Vafa RP, et al. Ibuprofen differentially affects supraspinatus muscle and tendon adaptations to exercise in a rat model. Am J Sports Med. 2016;44(9):2237-2245.
Drug Design, Development and Therapy

\section{Publish your work in this journal}

Drug Design, Development and Therapy is an international, peerreviewed open-access journal that spans the spectrum of drug design and development through to clinical applications. Clinical outcomes, patient safety, and programs for the development and effective, safe, and sustained use of medicines are the features of the journal, which

\section{Dovepress}

has also been accepted for indexing on PubMed Central. The manuscript management system is completely online and includes a very quick and fair peer-review system, which is all easy to use. Visit http://www.dovepress.com/testimonials.php to read real quotes from published authors. 